\title{
Hierarchical Automatic Differentiation by Vertex Elimination and Source Transformation
}

\author{
Mohamed Tadjouddinc ${ }^{1}$, Shaun A. Forth ${ }^{1}$, and John D. Pryce ${ }^{2}$ \\ 1 Applied Mathematics \& Operational Research, ESD \\ Cranfield University (RMCS Shrivenham), Swindon SN6 8LA, UK \\ \{M.Tadjouddine, S.A.Forth\}Ormcs.cranfield.ac.uk \\ 2 Computer Information Systems Engineering, DOIS \\ Cranfield University (RMCS Shrivenham), Swindon SN6 8LA, UK \\ J.D.Pryce@rmcs.cranfield.ac.uk
}

\begin{abstract}
We present a hierarchical scheme to extend the applicability of automatic differentiation (AD) by vertex elimination from the basic block level to code with branches and subroutine calls. We introduce the ELIAD tool that implements our scheme. Results from computational fluid dynamics (CFD) flux linearisations show runtime speedup by a typical factor of two over both finite-differencing and traditional forward and reverse modes of $A D$.
\end{abstract}

\section{Introduction}

In scnsitivity analysis, design optimisation or Newton solvers, we frequently need to compute derivatives of a function represented by a computer program. Finitcdifferencing is a popular way of calculating derivatives. It is easy to implement but incurs truncation errors that may affect robustness of algorithms that use derivatives. An alternative is to use the algorithms and tools of Automatic Differentiation (AD) [1-3], by which derivatives of a function represented by a computer program are computed without the truncation errors associated with finite differences.

The most efficient way to implement AD in terms of run-time speed is usually source transformation; here the original code is augmented by statements that calculate the needed derivatives. ADIFOR [4] and TAMC [5] are well-established tools for this which make use of the standard forward and reverse modes of $\mathrm{AD}$. The forward mode propagates directional derivatives along the flow of the program. The reverse mode first computes the function, then calculates the sensitivities of the dependent variables with respect to the intermediate and independent variables in the reverse order to their calculation in the function. The sensitivities of the dependent to the independent variables give the desired derivatives.

ELIAD [6-8] is an AD tool also using source transformation but, in contrast to the AD tools listed above, ELIAD uses the vertex climination algorithm of Griewank and Reese [9]. The Jacobian code created requires fewer floating-point 
operations than that obtained by the traditional forward and reverse AD methods as implemented by ADIFOR or TAMC. The vertex climination AD algorithm efficiently exploits the sparsity of the Jacobian calculation. Careful experiments showed ELIAD produced Jacobian codes running 2 to 10 times faster than those by ADIFOR or TAMC, see $[6,8]$.

Section 2 describes AD using the vertex elimination approach. Section 3 presents the source transformation approach and describes the ELIAD tool. Section 4 discusses the hierarchical vertex elimination approach as an efficient way of dealing with branches. Section 5 presents numerical results from CFD flux calculations and Sect. 6 concludes.

\section{AD by Vertex Elimination}

Following [2], we consider a computer program that represents a function $\mathbf{F}$ : $\mathbb{R}^{n} \rightarrow \mathbb{R}^{m}$. An execution of that computer program can be vicwed as a scquence of $p+m$ scalar assignments described as follows:

$$
x_{i}=\phi_{i}\left(\left\{x_{j}\right\}_{j \prec i}\right), \quad i=n+1, \ldots, N=n+p+m,
$$

where $\phi_{i}$ represents an elemental function and $j \prec i$ means that $x_{j}$ is used in computing $x_{i}$. We define the independent variables to be thosc input variables with respect to which we need to compute the derivatives, the dependent variables to be those outputs whose derivatives are desired, and the intermediate variables to be those whose value depends on an independent and affects a dependent variable. Further, we define an active variable to be an independent, intermediate or dependent variable. Without loss of generality, we assume that in (1), $x_{1}, \ldots, x_{n}$ are the independents, $x_{n+1}, \ldots, x_{n+p}$ are the intermediates, and $x_{n+p+1}, \ldots, x_{n+p+m}$ are the dependents and no dependent variable is calculated directly from another dependent variable.

The sequence of assignments in (1) yields the following non-lincar system,

$$
0=\left(\phi_{i}\left(\left\{x_{j}\right\}_{j \prec i}\right)-x_{i}\right), \quad i=n+1, \ldots, N .
$$

Assuming the $\phi_{i}$ have continuous first derivatives, we can differentiate the nonlincar system (2). Writing $\nabla x_{i}=\left(\frac{\partial x_{i}}{\partial x_{1}}, \ldots, \frac{\partial x_{i}}{\partial x_{n}}\right)$, we obtain

$$
0=\nabla \phi_{i}\left(\left\{x_{j}\right\}_{j \prec i}\right)-\nabla x_{i}=\sum_{j \prec i} \frac{\partial \phi_{i}}{\partial x_{j}} \nabla x_{j}-\nabla x_{i}, \quad i=n+1, \ldots, N \text {. }
$$

In matrix terms, denoting $c_{i, j}=\left\{\begin{array}{ll}\frac{\partial \phi_{i}}{\partial x_{j}} & n+1 \leq i, j \leq N \\ 0 & 1 \leq i, j \leq n\end{array}\right.$, and $\mathbf{C}=\left(c_{i, j}\right)_{1 \leq i, j \leq N}$, the linear system (3) can be rewritten as,

$$
\left(\mathbf{C}-\mathbf{I}_{N}\right) \nabla \mathbf{x}=\left[\begin{array}{l}
-\mathbf{I}_{n} \\
\mathbf{0}_{(p+m) \times n}
\end{array}\right]
$$


where $\mathbf{I}_{k}$ denotes the $k \times k$ identity matrix. The lower triangular matrix $\mathbf{C}-\mathbf{I}_{N}$ is called the extended Jacobian. The equivalent graph of the cxtended Jacobian matrix is called the computational graph (see the example of [8] for details). Adopting the standard notation [2, p. 161], the strictly lower triangular $N \times N$ matrix $\mathbf{C}$ can be written in block form, (with $\mathbf{L}$ strictly lower triangular),

$$
\mathbf{C}=\left[\begin{array}{lll}
0 & 0 & 0 \\
\mathbf{B} & \mathbf{L} & 0 \\
\mathbf{R} & \mathbf{T} & 0
\end{array}\right] .
$$

The Jacobian $\nabla \mathbf{F}$ of the function $\mathbf{F}$ is then the Schur complement of $\mathbf{R}$ in $\mathbf{C}-\mathbf{I}_{N}$ and can be calculated using some form of Gaussian elimination [2]. We use pivot orderings based on heuristics from sparse matrix technology such as the Markowitz critcrion studicd in $[2,9,10]$. Such heuristics aim to minimise the number of floating point operations at each step of the elimination.

\section{AD by Source Transformation}

An $\mathrm{AD}$ tool can be implemented using operator overloading or source transformation (see [2, chap. 5]). Automatic Differentiation by source transformation uses compiler techniques to parse and analyse the original code, then augments the code's statements that calculate real valucd variables with additional statements to calculate their derivatives.

\subsection{Source Transformation Approach}

As described in [11], a source transformation AD tool diffcrentiates a program in the following stages:

1. The input program is parsed into an abstract syntax tree, see [12], as in the front-end of a compiler (lexing, parsing, scmantic analysis).

2. The abstract syntax tree may be transformed into a semantically equivalent intermediate representation suited to applying the AD technique, e.g. see Sect. 4.2.

3. A dependency analysis is performed to determine intermediate variables from user defined independent and dependent variables.

4. The intermediate representation is augmented with derivative calculation statements.

5. Some optimisations $[12,13]$ (simplification of algebraic expressions, loop optimisations, statement rcordcring) are performed on the derivative code.

6. The transformed program is output in the source languagc.

\subsection{The EliAD Tool}

ELIAD is an AD tool for a restricted class of Fortran programs motivated by application to numerical flux evaluation in CFD. Such subroutincs typically have 
10 to 100 inputs and outputs, some hundreds of intermediate values, branches and, (assumed unrollable) loops. ELIAD is written in Java and uses a front-end and back-end gencratcd by ANTLR [14]. As depicted in Fig. 1, EliAD enables the user to build up the computational graph of an input code, save it as a file, and send it to a tool for finding elimination sequences, which we term the sequencer. A filc containing the elimination scquence is passed back to ELIAD, which then generates the corresponding derivative code. The representations of the computational graph and the sequence are described in [15]. The sequencer is currently a scparate Matlab program but will cventually be coded in Java to facilitate communication with ELIAD. Nonctheless, ELIAD provides two default sequences that are the forward and reverse orderings of the intermediate vertices.

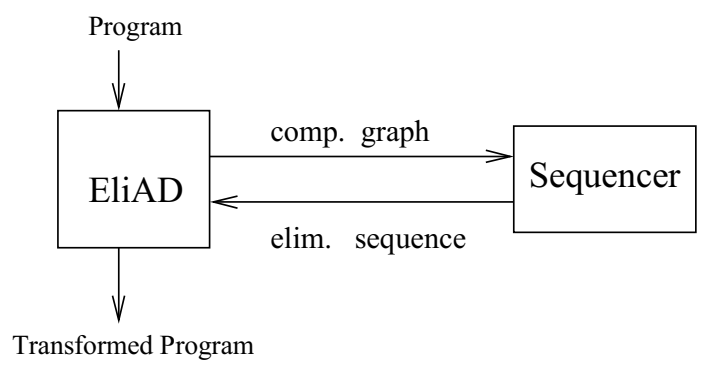

Fig. 1. EliAD framework

Building the Computational Graph The ELIAD front-end parses an input program into its abstract syntax trec. In a program transformation framework, the abstract syntax trec is usually processed to construct control or data flow graphs. We use a graph that we term the Abstract Computational Graph (ACG). This can be viewed as a control flow graph in which each basic block is expanded to a computational graph. Unlike the computational graph used in AD by opcrator overloading and that represents one cxecution of the program, the ACG takes into account all possible execution paths for all possible values of independent variables. For the restricted class of codes defined in Sect. 3.2, the ACG is a directed acyclic graph describing the chain of operations from the independent to the dependent variables.

A vertex of the ACG represents an active program variable, that is, a variable whose derivative value is not a priori zero. The independent[dependent] vertices are the ones that have no predccessors[successors]. An edge of the ACG, say from vertex $j$ to $i$, represents the dependency $j \prec i$ (defined in Sect. 2) and is labelled by the symbolic expression for local partial derivative $\frac{\partial x_{i}}{\partial x_{j}}$.

Generating the Derivative Code To generate a derivative code, EliAD is given a filc containing an elimination sequence provided by the sequencer of Fig. 1. Using the abstract syntax tree of the input code and the information kept 
in the abstract computational graph, the original code is interspersed with assignments that compute local partial derivatives. Then the elimination sequence is used to climinate intermediate vertices until the computational graph becomes bipartite. A vertex is eliminated by connecting each of its predecessors to all its successors. New edges are labelled by the product of existing edges linking the corresponding successors and predecessors. If the edge already exists, its label is updated by adding the product. This results in a series of scalar assignments that compute new entries or update existing entries of the extended Jacobian.

A difficulty in using the vertex climination approach in the AD source transformation framework is dcaling with branching. We use a hicrarchical climination scheme similar to [16] to overcome this difficulty.

\section{Hierarchical Vertex Elimination}

An IF block construct is viewed as a supcrnode of the ACG whose inputs and outputs are detcrmined using a read/write analysis. In the overall climination process, computational graphs within the supernodes are first rendered bipartite subgraphs whose vertices can then be climinated along with the remaining intermediate vertices to complete the Jacobian accumulation.

\subsection{Elimination of Supernodes}
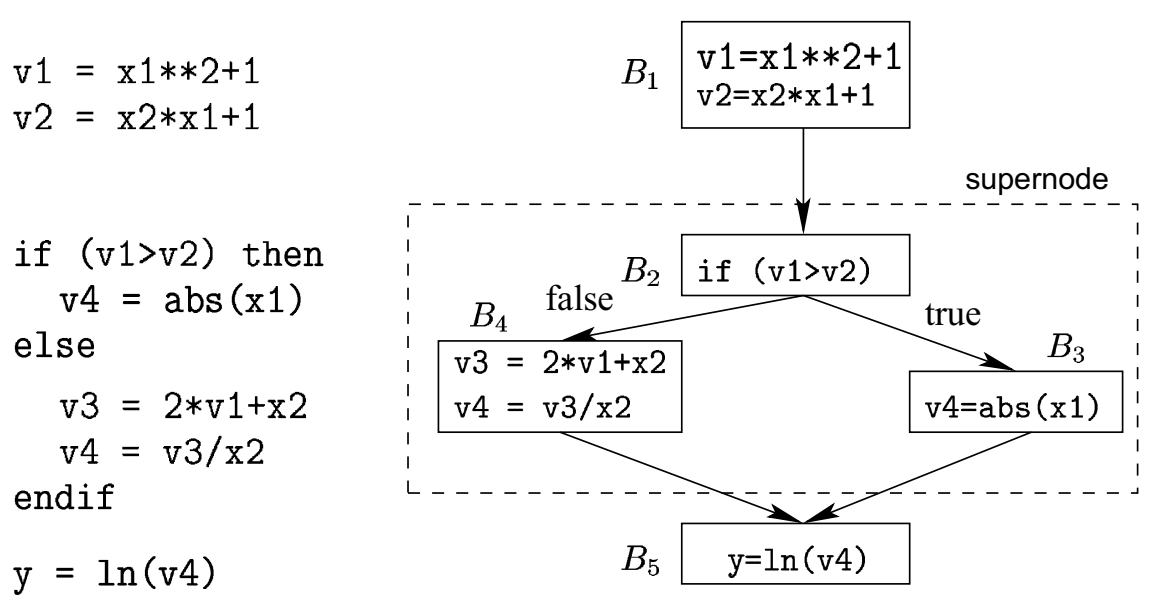

Fig. 2. Fortran code (left) and control flow graph (right)

Figure 2 shows a code fragment with an IF construct, and its flow graph. In the ACG, the basic blocks $B_{1}, B_{3}, B_{4}, B_{5}$ are expanded to their computational graph. Figurc 3 shows the computational graph of the basic block $B_{4}$. Each basic block is analysed to determine its inputs and outputs. The inputs are 


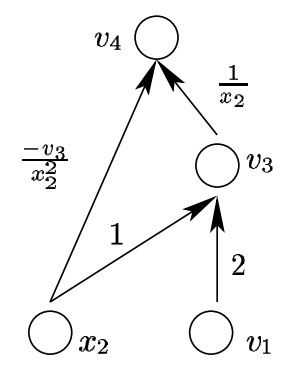

Fig. 3. The computational graph of the block $B_{4}$

those active variables that are written (assigned to) before, and read (appear on the right hand side of an assignment) within, the basic block. The outputs are those active variables that are written in the basic block and read thercafter. The inputs [outputs] of the IF construct (supernode) are the union of inputs [outputs] of its branches. This analysis corrects that of [17].

The elimination of a supernode takes three logical stages that wo describe by reference to Fig. 2.

1. The partial derivatives of the outputs of $B_{3}\left[B_{4}\right]$ with respect to the inputs of $B_{4}\left[B_{3}\right]$ are initialised to zero.

2. Local partial derivatives in $B_{3}$ and $B_{4}$ are calculated and generated at statcment level.

3. All intermediate vertices of $B_{3}$ and $B_{4}$ are eliminated and resulting statements are generated.

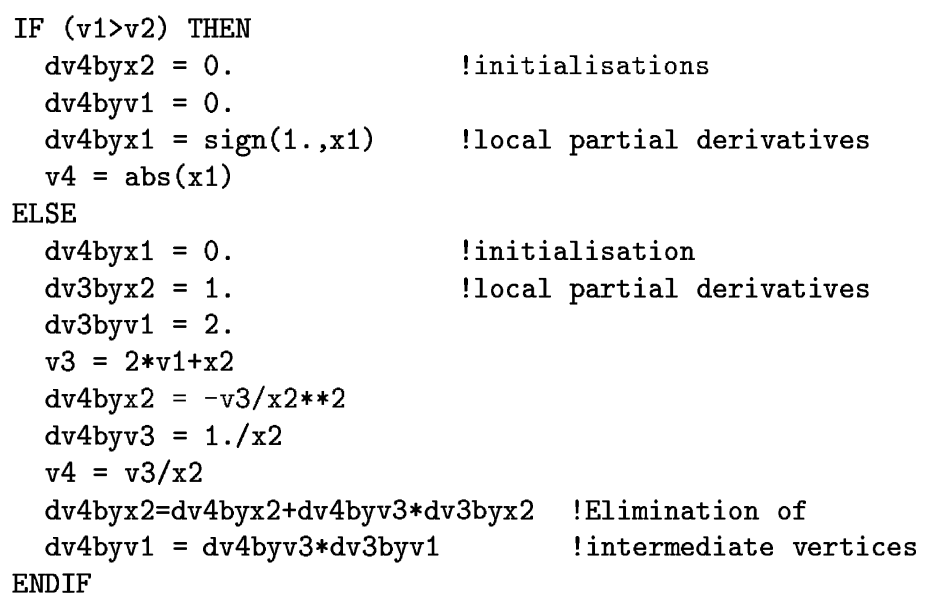

Fig. 4. Elimination of the supernode

This augments $B_{3}$ and $B_{4}$ with new statements that initialise or calculate derivatives and reduces the subgraphs within the supernode to a bipartite graph be- 
The analysis uses the wcll-known call graph, which is a directed graph representing the calling relationships between the procedures of the program [13]. A construction algorithm for the call graph can be found in [13].

The call graph is traversed bottom up to perform dependency and activity analyses for each subroutinc. Again, this is donc hicrarchically starting from the lowest level and recursively combining the analyses at a higher level. Finally, the call graph is traversed top down to take into account the context and consequently refine the analyses. This interprocedural analysis allows us to avoid calculating derivatives that can be identified a priori as being always zero.

For each subroutine, the analyses are performed using its control flow graph and result in:

- A dependency graph which shows the dependencies of each subroutine variable with respect to the subroutinc inputs.

- An activity graph which shows the set of active variables associated with each basic block of the control flow graph of the subroutine.

Both graphs arc used to construct the ACG for each subroutine as described in Sect. 3.2. The resulting ACG is then used to generate the corresponding derivative code as in Sect. 3.2. The ACG supernode associated to a subroutine call is built up by connecting the vertices representing its active outputs to those of its active inputs. The supernode edges are labelled by the corresponding components of the local Jacobian resulting from the differentiation of that called subroutine.

\section{$5 \quad$ Numerical Results}

In [7], the application of ELIAD to flux calculations in a finite-volume Parabolised Navier-Stokes space-marched flow-solver ensured quadratic convergence for the associated Newton solver and increased overall performance compared with traditional forward or reverse AD or sparse finite-differencing for Jacobian assembly.

Table 1 shows the ratio of CPU time for computing Jacobian $\nabla F$ to that for computing the function $F$ (with $n$ independents and $m$ dependents), for (a) the Roe flux with MUSCL interpolation $(n=20, m=5)$, (b) the Vigneron Flux $(n=5, m=8)$ and (c) the viscous flux $(n=10, m=4)$. The runs are from a COMPAQ Alpha DS20E workstation with $667 \mathrm{MHz}$ CPU, $128 \mathrm{~KB}$ L1-Cache and 8 MB L2-cache. The Roe flux code contains branches (Harten's entropy fix). All calculations were performed for 10,000 random sets of inputs repeated 10 and 50 times respectively. We used the forward and reverse modes of TAMC, 1-sided finite-differencing and ELIAD with Markowitz ordcring. In Table 1, timings in brackets are obtained with the TAMC's "-pure" option, which removes function calculation. This saves some floating-point opcrations and a similar capability will be developed for ELIAD. The ELIAD generated code is twice as fast as the next fastest $\mathrm{AD}$ technique (TAMC reverse) for Roe, (TAMC forward) for Vigneron and (TAMC reverse) for viscous fluxes. The TAMC(reverse) is slower for the Vigneron flux as it propagates 8 directional derivatives instead of 5 for 
Table 1. Efficiency of flux Jacobian calculations

(a) Roe flux, $\mathrm{CPU}(F)=7.31 \times 10^{-7} \mathrm{~s}$

\begin{tabular}{lcc}
\hline technique & $\mathrm{CPU}(\nabla F) / \mathrm{CPU}(F)$ & $\operatorname{Error}(\nabla F)$ \\
\hline TAMC(forward) & $18.5(18.3)$ & 0.0 \\
TAMC(reverse) & $9.1(7.6)$ & $3.6 \times 10^{-16}$ \\
Finite Difference & 24.1 & $4.0 \times 10^{-6}$ \\
ELIAD & 4.7 & $5.6 \times 10^{-15}$ \\
\hline
\end{tabular}

(b) Vigneron flux $\mathrm{CPU}(F)=1.93 \times 10^{-7} \mathrm{~s}$

\begin{tabular}{lcc}
\hline technique & $\mathrm{CPU}(\nabla F) / \mathrm{CPU}(F)$ & $\operatorname{Error}(\nabla F)$ \\
\hline TAMC(forward) & $3.8(3.0)$ & 0.0 \\
TAMC(reverse) & $8.9(7.5)$ & $4.4 \times 10^{-16}$ \\
Finite Difference & 8.3 & $1.3 \times 10^{-6}$ \\
ELIAD & 2.2 & $4.4 \times 10^{-16}$ \\
\hline
\end{tabular}

(c) Viscous flux Jacobian, CPU $(F)=4.22 \times 10^{-7} s$

\begin{tabular}{lcc}
\hline technique & $\mathrm{CPU}(\nabla F) / \mathrm{CPU}(F)$ & $\operatorname{Error}(\nabla F)$ \\
\hline TAMC(forward) & $5.8(5.4)$ & 0.0 \\
TAMC(reverse) & $3.6(3.6)$ & 0.0 \\
Finite Difference & 13.4 & $4.5 \times 10^{-8}$ \\
ELIAD & 1.8 & $5.6 \times 10^{-17}$ \\
\hline
\end{tabular}

TAMC(forward). We also show the maximum error in the entries of the Jacobians, assuming TAMC(forward) is exact. The ELIAD Jacobians are correct to machine precision. The crror in the 1-sided finite-difference approximation is in line with its truncation error.

\section{Conclusions}

We have described a hicrarchical vertex elimination approach that efficiently deals with branches and subroutine calls. This approach may be seen as an extension of the hierarchical approach of [16] from the subroutine level to the IF block level. It allows us to systematically generate Jacobian code valid for all possible execution paths, that is near optimal within each level of the hierarchy.

We have implemented this approach in the ELIAD tool. For the Roe code which contains branches and other non-branching CFD codes, the ELIAD generated Jacobian code executes about twice as fast as that generated using traditional forward and reverse mode AD tools. This run-time improvement is mainly duc to the full sparsity exploitation enabled by the elimination approach.

An ongoing difficulty is that the computational graph construction requires static analyses that are in gencral conscrvative. Generally, safe overestimations are used to ensure correctness and efficiency [18]. Typically, loops require array region analyses to maintain the efficiency of this approach. Future work will investigate tcchniques that deal with loops within our framework. 


\section{Acknowledgments}

We thank EPSRC and UK MOD for funding this project under grant GR/R21882.

\section{References}

1. Griewank, A., Corliss, G.: Automatic Differentiation of Algorithms. SIAM, Philadelphia (1991)

2. Griewank, A.: Evaluating Derivatives: Principles and Techniques of Algorithmic Differentiation. SIAM, Philadelphia (2000)

3. Corliss, G., Faure, C., Griewank, A., Hascoët, L., Naumann, U., eds.: Automatic Differentiation of Algorithms: From Simulation to Optimization. Springer (2002)

4. Bischof, C., Carle, A., Khademi, P., Mauer, A.: ADIFOR 2.0: Automatic differentiation of Fortran 77 programs. IEEE Computational Science \& Engineering 3 (1996) 18-32

5. Giering, R., Kaminski, T.: Recipes for Adjoint Code Construction. ACM Trans. on Math. Software 24 (1998) 437-474

6. Forth, S.A., Tadjouddine, M., Pryce, J.D., Reid, J.K.: Jacobian Code Generated by Source Transformation and Vertex Elimination is as Efficient as Hand-coding. ACM Trans. on Math. Software (2002) (submitted).

7. Forth, S.A., Tadjouddine, M.: CFD Newton Solvers with EliAD, An Elimination Automatic Differentiation Tool. In: Int. Conf. on CFD. Springer-Verlag, Sydney (2003) (to appear).

8. Tadjouddine, M., Forth, S.A., Pryce, J.D., Reid, J.K.: Performance Issues for Vertex Elimination Methods in Computing Jacobians using Automatic Differentiation. In Sloot, P.M., Tan, C.K., Dongarra, J.J., Hoekstra, A.G., eds.: ICCS, Part II. Volume 2330 of LNCS. Springer, Amsterdam (2002) 1077-1086

9. Griewank, A., Reese, S.: On the calculation of Jacobian matrices by the Markowitz rule. [1] 126-135

10. Naumann, U.: Elimination techniques for cheap Jacobians. [3] 241-246

11. Bischof, C., Roh, L., Mauer, A.: ADIC - An Extensible Automatic Differentiation Tool for ANSI-C. Software-Practice and Experience 27 (1997) 1427-1456

12. Aho, A., Sethi, R., Ullman, J.: Compilers: principles, techniques, and tools. Addison-Wesley (1986)

13. Zima, H., Chapman, B.: Supercompilers for Parallel and Vector Computers. Addison-Wesley Publishing Company (1991)

14. Parr, T., Lilly, J., Wells, P., Klaren, R., Illouz, M., Mitchell, J., Stanchfield, S., Coker, J., Zukowski, M., Flack, C.: ANTLR Reference Manual. Technical report, MageLang Institute's jGuru.com (2001) See http://www.antlr.org/doc/.

15. Naumann, U., Forth, S.A., Tadjouddine, M., Pryce, J.D.: A Standard Interface for Elimination Sequences in Automatic Differentiation (Version 1). AMOR Report 01/3, Cranfield University (RMCS Shrivenham), Swindon SN6 8LA, UK (2001)

16. Bischof, C.H., Haghighat, M.R.: Hierarchical approaches to automatic differentiation. In Berz, M., Bischof, C., Corliss, G., Griewank, A., eds.: Computational Differentiation: Tech., Appli., and Tools. SIAM, Philadelphia, Penn. (1996) 83-94

17. Tadjouddine, M., Forth, S.A., Pryce, J.D.: AD tools and prospects for optimal AD in CFD flux Jacobian calculations. [3] 247-252

18. Creusillet, B., Irigoin, F.: Exact vs. Approximate Array Region Analyses. In Sehr, D.C., Banerjee, U., Gelernter, D., Nicolau, A., Padua, D.A., eds.: Lang. and Comp. for Parallel Computing. Volume 1239 of LNCS. Springer (1997) 86-100 